\title{
Evaluation of a Pre-clinic Diabetes Assessment and Mapped Care Planning Intervention: a Multi-Centre Randomised Controlled Trial
}

Katharine Katharine Barnard-Kelly ( $\sim$ katharinebarnard@bhrltd.com )

Southern Health NHS Foundation Trust https://orcid.org/0000-0002-3888-3123

Ryan Charles Kelly

Spotlight-AQ

Peter Phiri

Southern Health NHS Foundation Trust

Hermione Price

Southern Health NHS Foundation Trust

Amar Ali

Blackburn and Darwen CCG

Irene Stratton

Gloucestershire Hospitals NHS Foundation Trust

Kayleigh Austin

Southern Health NHS Foundation Trust

Alice Neave

Southern Health NHS Foundation Trust

\section{Research Article}

Keywords:

Posted Date: February 3rd, 2022

DOI: https://doi.org/10.21203/rs.3.rs-1030575/v1

License: (1) (1) This work is licensed under a Creative Commons Attribution 4.0 International License. Read Full License 


\section{Abstract}

Introduction: Existing therapeutic interventions to treat diabetes are well known, yet the majority of people with diabetes do not consistently achieve blood glucose targets (even individual therapy targets) for optimal health, despite the large range of treatment options available. Such outcomes have remained stubbornly poor for decades with $<25 \%$ adults with diabetes achieving glycaemic targets. Patient behaviour, individually supported in routine clinical care, is an important missing component to improved outcomes, in a medical healthcare model not ideally suited to supporting successful diabetes management.

Participants: Adults with type 1, type 2 or pre-diabetes attending routine care outpatient appointments.

Design: A multi-centre, parallel group, individually randomised trial comparing consultation duration in adults with type 1, type 2 or pre-diabetes using the Spotlight Consultations pre-clinic assessment compared to usual care in the Spotlight-AQ study.

Intervention: An outpatient pre-clinic intervention delivered within one week prior to scheduled routine outpatient appointment.

Sample size: 200 recruited across up to 10 sites.

Primary outcome measure: Duration of routine outpatient consultation.

\section{Secondary outcome measures:}

Functional health status

diabetes distress

depression

treatment satisfaction

impact on self-care behaviours

HCP burnout

HCP treatment satisfaction and burden

hypoglycaemia (time less than $70 \mathrm{mg} / \mathrm{dL}$ )

hyperglycaemia (time above $180 \mathrm{mg} / \mathrm{dL}$ )

Change in weight

Change in $\mathrm{HbA}_{1 \mathrm{c}}$ 
Ethics and dissemination: The trial was approved by the Wales REC7 Research Ethics Committee (21/WA/0020). Results will be disseminated through national and international conferences, scientific journals, newsletters, magazines and social media. Target audiences include consultants and other clinicians in diabetes, and medical professionals or scientists overall.

\section{Trial Registration: ISRCTN15511689}

\section{Introduction}

There is an urgent need to develop interventions that lead to sustained improvements in glycaemic and quality of life outcomes for people with diabetes and that support diabetes prevention. Therapeutic interventions to treat diabetes have been shown to be effective in clinical trials, yet $>75 \%$ of people with diabetes consistently do not achieve blood glucose targets (even individual therapy targets) for optimal health, despite the large variety of drugs, including insulin, and medical devices available e.g. insulin pumps [1]. Such glycaemic outcomes have remained stubbornly poor for decades and is a significant risk factor for microvascular and macrovascular outcomes. Patient behaviour, individually supported in routine clinical care, is an important missing component to improved outcomes, in a medical healthcare model poorly suited to supporting successful diabetes management.

As such, it is important to recognize the different needs of each individual and support skills development so that individuals are empowered to undertake effective disease self-management. Data from the International Diabetes Federation (IDF) shows that $10 \%$ of global health expenditure is spent on diabetes (US $\$ 760$ billion), predicted to rise to $\$ 825 \mathrm{bn}$ by 2030 [2]. Indirect costs from premature death, disability and other health complications due to diabetes are estimated to add an additional $35 \%$ to the annual global health expenditure associated with the condition [2]. The intangible costs, however are less visible but include worry, anxiety, discomfort, pain, loss of independence, concerns about managing the condition, fears for future complications and their potential impact on quality of life. These are also significant contributors to the cost of diabetes.

Depression is commonly reported to be 2-3 times more prevalent in people with diabetes than the general population [3]. This figure perhaps overshadows the significant number of individuals who do not report symptoms of depression, but experience diabetes distress. These emotions can be described as: feeling overwhelmed and defeated by diabetes; feeling angry about diabetes, frustrated by the self-care regimen and/or having strong negative feelings about diabetes; feeling that diabetes is controlling their life; worrying about not taking care of diabetes well enough, yet unable, unmotivated or unwilling to change; avoiding diabetes-related tasks that give feedback about consequences of poor control; and/or feeling alone/isolated with diabetes. In type 2 diabetes, distress (but not depression) was related with poor glycaemic control and change in distress (but not change in depressive symptoms) was associated with both short- and long-term change in glycaemic control [4]. Similar relationships were found in T1D: diabetes-specific emotional distress (measured by the DDS) was related to glycaemic control in a 
Norwegian study and was also linked to worsening diabetes management over time in adults with T1D [5].

Routine patient care visits currently leave both patients and healthcare professionals feeling frustrated both in primary and specialist care settings. The lack of understanding of the psychosocial burden of diabetes and the evolving consequences results in a negative impact on clinical practice with consequential negative outcomes for patients and increasing frustration for healthcare professionals. Complex and detailed algorithms are supplied by various guidelines for the management of blood glucose, lipids, blood pressure and long-term complications, but these relate only to medical management. Even goals which have been mutually agreed upon are often not followed up, leaving patients frustrated and healthcare professionals struggling to provide tailored support. Typically, physicians interrupt their patients 11 seconds after they start describing their problems; approximately half of patients' concerns are not discussed, and in half of health care visits, patients and physicians disagree on the central problem presented [6]. Disagreement about treatment goals, inconsistency among healthcare teams and confusion about treatment priorities are associated with poorer outcomes [7].

Burnout amongst healthcare professionals is a key challenge affecting healthcare practice, safety and quality of care. It is estimated that more than half of US physicians experience substantial symptoms of burnout, with burnout almost twice as prevalent among physicians as US workers in other fields [8]. Nurses experience a similarly high prevalence of burnout and depression, with $43 \%$ reporting high degrees of emotional exhaustion. COVID has exacerbated this problem. Furthermore, there are significant correlations between a physician's sense of depersonalisation and patient satisfaction with their hospital care, and between a physician's job satisfaction and patient satisfaction with their healthcare and patientreported adherence to medical advice.

\section{Study Design}

Spotlight is a multicentre, parallel group, individually randomised RCT. It will compare consultation length, biomedical and psychosocial outcomes in adults with type 1, type 2 or pre-diabetes attending routine outpatient appointments for their diabetes care.

\section{Eligibility}

\subsection{Inclusion Criteria}

- $\geq 18$ years. There is no upper age limit

- Diagnosed with T1D or at risk of or diagnosed with T2D (including pre-diabetes) for at least 6 months

- Any diabetes treatment

- Willing/able to use Spotlight Consultations tool 
- Ability to give informed consent

- Ability to speak and read English fluently

\subsection{Exclusion Criteria}

- $<18$ years

- Mental illness that could seriously reduce their ability to participate in the study

- Lack of capacity

\section{Recruitment}

\subsection{Screening and Consent}

Outpatient diabetes surgery appointment clinic lists will be scrutinised ahead of appointments and for people who appear eligible. An information sheet explaining the trial will be sent by post or email as appropriate (including contact details to opt out if the person does not want to be contacted about the trial). Before the outpatient appointment a member of the research team will contact the prospective participant to discuss the study at least 24 hours before the outpatient appointment allowing sufficient time for reflection and discussion. This will allow those who are eligible for the study to be randomised immediately after the outpatient appointment. If the participant wishes to take part, they will have the opportunity to discuss the study face-to-face with a research nurse before they give written consent. Final eligibility criteria will be checked before the participant is recruited. Patients whose medical records cannot be accessed prior to the appointment to determine eligibility (e.g. patients from another hospital), will be given information about the study on the day of their outpatient appointment and will be offered the opportunity to come on another day to discuss their participation in the trial.

\subsection{Randomisation}

Once the consent process has been completed study participants will be randomized into either the intervention group or control group for 12 months. We will randomise on a 1:1 basis using computerised randomisation software.

Those randomised to the intervention group will be asked to complete study questionnaires and the Spotlight Consultation pre-clinic assessment. The results of the Spotlight Consultation digital health assessment will be discussed between the participant and healthcare professional during the outpatient visit along with identified matched care pathways and agreement made on best-fit action plan.

\section{The Spotlight Consultations Intervention}




\subsection{Intervention Arm}

Participants who are randomised to receive the Spotlight Consultations intervention will be sent a unique secure link to the Spotlight-AQ platform.

Telemedicine or In Clinic Visits: Participants will have a telemedicine or in-person clinic visit as per their usual routine care during the trial. Study participants will complete a personal assessment on study tablet devices if face-to-face visit. The support of a research assistant will be available if required. HCPs will also access the results and care pathway options, via their own secure portal logins. HCPs and participants will discuss the priorities and possible options collaboratively in a co-decision making, person-centred approach. Participants will be invited for repeat assessment as per routine care.

\subsection{Control Arm}

Participants in the control group will continue to receive usual care. Control group participants will be offered access to the Spotlight platform and pre-clinic assessment at the end of the RCT, for use in their next schedule routine outpatient appointment.

\subsection{Follow Up}

After the participants are randomised to either the intervention or control arm, data will be collected from them at the following timepoints:

- Pre scheduled routine outpatient appointment (baseline)

- Three, six, nine and twelve months post visit

Types of data that will be collected, in addition to the baseline questionnaires that will be repeated, will be:

- Mortality

- Adverse events

- Health economic questions

Healthcare professional questionnaire data will be collected at baseline, six and twelve months. Key healthcare professionals involved in the delivery of the intervention will be interviewed once around 12 months after the start of the trial in their centre.

\subsection{Qualitative Evaluation}

The aims of the qualitative evaluation are to understand and explore: 
- Participants' experiences, including gender specific experiences, of receiving Spotlight Consultations tool and health professionals' views about delivering it.

- The perceived benefits of Spotlight Consultations from participants' and health professionals' perspectives; and, their recommendations for future refinements.

- Any changes participants make to their diabetes self-management practices and treatment goals after receiving Spotlight Consultations intervention and why.

- Whether, in what ways and, why, Spotlight Consultations is delivered and received differently in different settings

- Whether there are any site-specific differences in how participants self-manage their diabetes after receiving Spotlight Consultations, and why.

\subsubsection{Participant interviews}

20 participants randomized to receive the intervention will be interviewed prior to receiving the intervention to explore expectations, reasons for participation and perceived facilitators and barriers to achieving treatment goals. The same participants will be re-interviewed 6 months later to look at whether, how and, why, their diabetes self-management practices and treatment goals have changed in the intervening 6 months; and, any perceived barriers to achieving future changes and goals. These interviews will explore their experiences of receiving Spotlight Consultations tool; any changes made to their diabetes self-management practices, and why; short- and long-term treatment goals and the reasons for these; and perceived barriers and facilitators to achieving these goals. These interviews will also include detailed exploration of participants' historical diabetes management practices; previous contact with health professionals and diabetes management programs; and, their everyday work and family lives. The interviews will also explore participants' information and support needs and whether, and in what ways, the intervention and follow-up care could be changed or improved.

\subsubsection{Health professional interviews}

Health professionals involved in Spotlight Consultations delivery will be interviewed once at the end of the trial. Interviews will explore: previous experiences of delivering self-management interventions for adults with diabetes; perceived benefits of Spotlight Consultations as compared to other interventions; experiences of, and views about, the training received to deliver Spotlight Consultations; barriers and facilitators to intervention delivery; perceived impact of Spotlight Consultations on participants' diabetes self-management practices; and, how Spotlight Consultations could be changed/improved for future use.

Qual Topic guides: Participant and health professional interviews will be informed by topic guides, with questioning kept sufficiently flexible to enable individual issues to be identified and explored. All interviews will be audio-recorded, transcribed in full, and early interviews will be reviewed by the research team to determine whether any alterations to the topic guides need to be made.

\subsubsection{Process Evaluation}


The process evaluation will be undertaken 'to explain discrepancies between expected and observed outcomes, to understand how context influences outcomes, and to provide insights to aid implementation'. Interviews will be held with a subgroup of participants $(n=6)$, healthcare professionals $(n=3)$ and clinical triallists $(n=2)$.

\section{Health Economic Analysis}

QALYs will be estimated from EQ-5D-5L and mortality data, using the area-under-the-curve method. Similarly, costs will be estimated at the patient level. Mean between-group differences in QALYs and costs will be estimated using a regression-based approach, including adjustment for baseline covariates and interaction terms for pre-defined sub-groups, and allowing for clustering at hospital and/or practitioner level. Results will be presented as an Incremental Cost-Effectiveness Ratio (ICER) if appropriate. Non-parametric bootstrapping will be used to estimate confidence intervals around estimated cost differences and ICERs.

A simple modelling approach will also be used to estimate the costs and health impacts of surgical complications over a lifetime horizon. This long extrapolation is necessary to reflect any mortality or lasting quality of life decrement associated with surgical complications. There will be no attempt to estimate the long-term impact of improved diabetes management related to the intervention, as it will be difficult to predict the duration over which any improvements will be maintained. This is likely to be a conservative assumption that will under-estimate the QALY gain and cost-effectiveness of intervention if it proves to be effective. Model parameters will be estimated from the trial and from other published sources. Long-term resource use, mortality and utility decrements associated with key surgical complications, will be identified by systematic review of HTAs, NICE guidelines and published literature.

\section{Statistics}

Demographics and characteristics of participants at baseline will be summarised and assessed for comparability between the intervention and control arms (16). The primary analysis will be conducted using ANCOVA adjusted for randomisation stratification factors on an intention to treat population. Continuous data will be presented as means and standard deviations and analysed using ANCOVA (or presented as medians and ranges and analysed using Mann-Whitney $U$ tests if data are skewed). Binary data will be reported in terms of odds ratios and analysed using logistic regression modelling. Analysis of time-to-event outcomes will include presenting Kaplan-Meier graphs by arm and analysed using Cox proportional hazards regression (or competing risk regression as discussed below). A two-sided $p$-value of 0.05 or less will be used to declare statistical significance for all analyses and results will be presented with $95 \%$ confidence intervals. Subgroups will be investigated, including those with $\mathrm{HbA}_{1 \mathrm{c}}$ above or below $69 \mathrm{mmol} / \mathrm{mol}$ at presentation; type of diabetes; age above or below 75 years. The cut-off of $69 \mathrm{mmol} / \mathrm{mol}$ has been chosen as the level above which the Joint British Diabetes Societies recommend specific action to improve pre-operative glycaemic control. 


\section{Strengths And Limitations Of The Study}

- Novelty of the intervention / the fact that it addresses an important gap

- Choice of outcome measures will enable a complete evaluation of both clinical outcomes and costeffectiveness

- Sample size and number of sites will make the results representative of a wide population and centres with differences in practice

- Intervention can be delivered face-to-face or remotely due to Covid and practical issues

- Covid-19 may create delays and recruitment problems

- Intervention requires use of tablet device (provided) however is unsuitable for adults with low literacy or poor English

\section{Declarations}

\section{ETHICS APPROVAL AND CONSENT TO PARTICIPATE}

Ethical approval was obtained by the Wales REC7 Research Ethics Committee (21/WA/0020). The study will be conducted in accordance with WMA Declaration of Helsinki and as revised and recognised by governing laws and EU Directives.

\section{CONSENT FOR PUBLICATION}

All participants have given / will sign informed consent for anonymised publication of data.

\section{AVAILABILITY OF DATA AND MATERIAL}

On request from the $\mathrm{PI}$.

\section{ACKNOWLEDGEMENTS}

We would like to thank Southern Health NHS Foundation Trust for their sponsorship.

\section{Author Contributions}

RCK, PP, AA, IS, KA, AN and KBK wrote the first draft of the protocol. HM refined the protocol. PP is the sponsor of the clinical trial, KBK the principal investigator and HP the chief medical officer. All authors critically reviewed the manuscript.

\section{Funding}

None

\section{Competing Interests}


Spotlight-AQ owns the pre-clinic assessment platform.

\section{TRIAL STATUS}

Protocol v. $1.10 ; 24^{\text {th }}$ March 2021

Recruitment start: 18 October 2021; recruitment completion: 31 March 2022

\section{References}

1. Foster NC, Beck RW, Miller KM, Clements MA, Rickels MR DiMeglio LA, Maahs DM, Tamborlane WV, Bergenstal R, Smith E, Olson BA, Garg SK. State of Type 1 Diabetes Management and Outcomes from the T1D Exchange in 2016-2018. Diabetes Technol Ther 2019 Feb;21(2):66-72. doi:

10.1089/dia.2018.0384. Epub 2019 Jan 18.

2. International Diabetes Federation Diabetes Atlas $9^{\text {th }}$ Edition, 2019. https://diabetesatlas.org/en/sections/individual-social-and-economic-impact.html last accessed 16th November 2020

3. Barnard K, Skinner T, Pevelar R. The prevalence of co-morbid depression in adults with type 1 diabetes: Systematic literature review. Diabet Med 2006; 23: 445-448. PMID: 16620276

4. Aikens JE. Prospective associations between emotional distress and poor outcomes in type 2 diabetes. Diabetes Care 2012; 35:2472-2478. PMID: 23033244

5. Lloyd CE, Pambianco G, Orchard TJ. Does diabetes-related distress explain the presence of depressive symptoms and/or poor self-care in individuals with type 1 diabetes? Diabet Med 2010; 27:234-237. PMID: 20546270

6. Singh Ospina, N., Phillips, K.A., Rodriguez-Gutierrez, R. et al. Eliciting the Patient's Agenda- Secondary Analysis of Recorded Clinical Encounters. J GEN INTERN MED 34, 36-40 (2019). https://doi.org/10.1007/s11606-018-4540-5

7. Barnard KD, Lloyd CE, Dyson PA, Davies MJ, O'Neil S, Naresh K, Lawton J, Ziegler R, Holt RI. Kaleidoscope model of diabetes care: time for a rethink? Diabet Med 2014; 31:522-530. PMID: 24506524

8. Reith TP. Burnout in United States Healthcare Professionals: A Narrative Review. Cureus 2018 Dec 4;10(2):e3681 